\title{
Distributed Collaboration for Enhanced Sender-Driven Video Streaming
}

\author{
Jacob Chakareski and Pascal Frossard, Senior Member, IEEE
}

\begin{abstract}
We propose a sender-driven system for adaptive streaming from multiple servers to a single receiver over separate network paths. The servers employ information in receiver feedbacks to estimate the available bandwidth on the paths and then compute appropriate transmission schedules for streaming media packets to the receiver based on the bandwidth estimates. An optimization framework is proposed that enables the senders to compute their transmission schedules in a distributed way, and yet to dynamically coordinate them over time such that the resulting video quality at the receiver is maximized. To reduce the computational complexity of the optimization framework an alternative technique based on packet classification is proposed. The substantial reduction in online complexity due to the resulting packet partitioning makes the technique suitable for practical implementations of adaptive and efficient distributed streaming systems. Simulations with internet network traces demonstrate that the proposed solution adapts effectively to bandwidth variations and packet loss. They show that the proposed streaming framework provides superior performance over a conventional distortion-agnostic scheme that performs proportional packet scheduling on the network paths according to their respective bandwidth values.
\end{abstract}

Index Terms-Channel coding, distributed collaboration, Internet, packet scheduling, source prunning, optimal control, packet partitioning, protocols, rate control, rate-distortion optimization, sender-driven transmission, video coding, video streaming.

\section{INTRODUCTION}

C OLLABORATIVE streaming has drawn considerable attention in recent years. One of the scenarios that fall into this category is distributed streaming, where multiple senders transmit packets over separate network paths to a single receiver. This setting can be encountered for example in Content Delivery Networks (CDNs), where multiple streaming or edge servers may stream multimedia data to a single client, or in peer-to-peer ( $\mathrm{P} 2 \mathrm{P})$ overlay networks, where a client may have access to the same multimedia data at multiple peers in the network. A related concept is the Digital Fountain model [1] where

Manuscript received March 17, 2006; revised January 16, 2008 and January 18, 2008. Published July 9, 2008 (projected). This work was presented in part at the International Conference on Multimedia and Expo (ICME), Toronto, Canada, July 2005. This work was supported in part by the Swiss National Science Foundation under Grants PP002-68737 and CTI 7388.2 ESPP-E. The associate editor coordinating the review of this manuscript and approving it for publication was Dr. Wenjun (Kevin) Zeng.

J. Chakareski was with the Signal Processing Institute, Ecole Polytechnique Fédérale de Lausanne (EPFL), 1015 Lausanne, Switzerland.

P. Frossard is with the Signal Processing Institute, Ecole Polytechnique Fédérale de Lausanne (EPFL), 1015 Lausanne, Switzerland (e-mail: pascal.frossard@epfl.ch).

Color versions of one or more of the figures in this paper are available online at http://ieeexplore.ieee.org.

Digital Object Identifier 10.1109/TMM.2008.921846 the system tries to minimize the download time of a file at a client by connecting to multiple mirror server sites.

The possibility to receive the same data over multiple paths increases the resilience of the media presentation to network outages or congestion onsets. These may occur on some of the paths and thereby may prevent the timely delivery of the data units sent exclusively over those individual paths. Furthermore, if the network paths exhibit good transmission quality it may be still desirable to spread the transmissions of the media data over multiple paths, i.e., to send different data units over different paths, in order to reduce the start-up delay of the client application and to ensure smooth and continuous play-out. The adaptive streaming session can be controlled either by the receivers, or by the senders. The latter solution provides several advantages in terms of media optimization, since the relative importance of the media packets can be known at the servers. In addition, the deployment of practical solutions is facilitated in this case since the management of overall network resources becomes easier, and the clients do not require any complex or non-standard functionality to access the streaming application.

We propose in this paper a distributed sender-driven streaming solution where servers collaboratively adapt to the network status in order to provide the media client with a superior video quality. The objective is to take benefit from the advantages offered by multipath streaming [2], [3] without complex synchronization or communication between servers. Instead of computing transmission schedules for every sender as in receiver-driven approaches, the client only monitors incoming packets and sends back information about the network availability. This information is distributed to all the servers, such that they can coordinate the delivery of the media stream, and avoid wasting bandwidth resources. Bandwidth estimates are then used in conjunction with a rate-distortion optimization framework to compute appropriate transmission actions at each sender. In essence, this framework enables the senders, based on the feedback information from the client, to compute independently, yet in a coordinated fashion, what their respective transmission policies should be, given the available bandwidth on each network path. In order to maximize the resulting video quality at the receiver, each sender takes into account the relative distortion importance of every video packet when computing its transmission policy.

The computational load imposed by the global optimization framework, however, increases with the number of servers, which may be prohibitive in practical scenarios. Therefore, we propose an alternative algorithm based on a priori packet classification. The technique achieves similar performance with a dramatically reduced complexity, thereby providing an 
interesting solution for practical and scalable implementations of adaptive and efficient distributed streaming systems. Simulation results on real internet traces demonstrate the superiority of the proposed rate-distortion optimized system compared to distortion-agnostic streaming solutions.

To the best of our knowledge, the earliest work that studied the problem of transmission coordination among the multiple senders in distributed streaming is [4]. In this work, the authors propose an algorithm that is run at the client and that performs rate allocation and packet partitioning among the senders. Based on this information the servers then adjust their sending rates in order to meet constraints from the network, which aggregated bandwidth still allows to transmit the complete media stream. In a follow-up work, the authors combined the previously proposed algorithm with forward error correction for improved error resilience to burst packet loss [5]. Similarly, the works in [6], [7] consider receiver-driven control protocols that synchronize the senders' transmissions in a rate-distortion optimized way. For improved error-resilience, Multiple Description Coding (MDC) is employed at each sender to pre-encode (prior to transmission) a progressively encoded media content that is streamed afterwards to the client. Another related work is [8], where a rate-distortion optimization framework is proposed for packet scheduling in receiver-driven distributed video streaming. The paper establishes that the gains in performance due to server (path) diversity, relative to a single server (path) case, are dependent on the quality of the network paths in terms of packet loss and delay. Finally, the works in [3], [9] examine the performance of an MDC scheme for distributed video streaming in CDNs. The authors report a $20-40 \%$ reduction in client video distortion, for the considered network conditions and topologies, relative to conventional CDNs that do not employ multiple description encoded video streams.

Differently from the prior work, we propose in this paper an optimization framework for sender-driven streaming, where multiple servers synchronize their transmission schedules for sending parts of a standard (single description) video stream under bandwidth and delay constraints. Each server selects the media packets that have to be transmitted in priority and determines the corresponding scheduling strategy in order to minimize the distortion at the receiver. The assistance from the receiver is limited to feedback necessary for bandwidth estimation, which is certainly required for the design of efficient adaptive streaming strategies.

The rest of the paper is organized as follows. In Section II, we describe the distributed streaming system, and formulate the rate-distortion optimization problem when servers collaborate to minimize the distortion at the client. Section III describes the media and network models, and the methodology that is used for computing the rate-distortion optimal packet scheduling strategy. A low-complexity solution is later proposed in Section IV, where a priori source pruning and packet classification allow to dramatically reduce the computational complexity relative to the original optimization framework. Finally, in Section V, simulation experiments on synthetic and realistic network traces show that both algorithms outperform a conventional distortion-agnostic system, which performs packet scheduling based only on the available bandwidth.

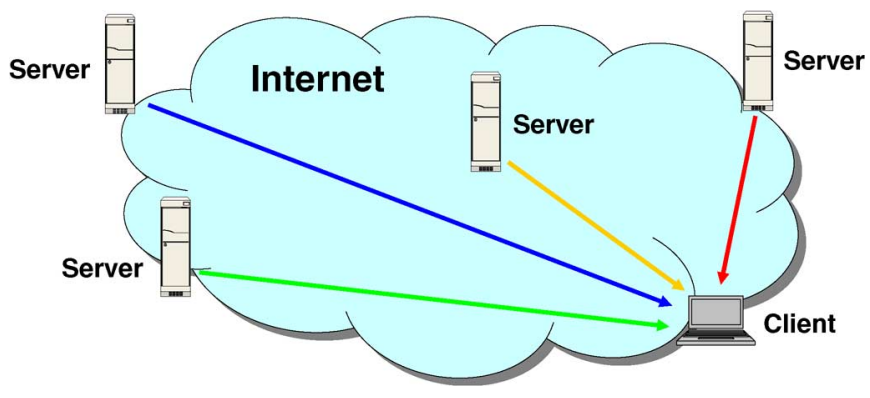

Fig. 1. Distributed streaming: multiple senders—single receiver.

\section{SENDER-DRIVEN DistRIBUTED STREAMING}

\section{A. Media Communication Model}

We consider a streaming system with $M$ senders (servers) transmitting a media presentation on $M$ independent bidirectional paths to a streaming media client, as illustrated in Fig. 1. The media presentation is available at each server in the form of a collection of interdependent data units with delivery deadlines. The servers encapsulate media data units into packets and collaboratively stream (parts of) the media presentation to the streaming client. The client, in turn, monitors incoming packets, and piggybacks information about the availability of the network paths on acknowledgment packets to all the sending servers. Based on this information, the servers periodically estimate the bandwidth available on all the paths from the senders to the media client. At the end of each estimation period $\Delta T$ the servers therefore know bandwidth estimates $\widetilde{R}_{1}, \ldots, \widetilde{R}_{M}$ for every path. This information is used to adapt the rate-distortion optimized streaming strategy to a varying network availability.

Based on bandwidth estimates, the servers then select the appropriate transmission schedules for sending the media packets. Specifically, a transmission schedule or policy represents the actions performed by the servers on a given data unit $l$, at each transmission opportunity. More formally, let $t_{0}, t_{1}, \ldots, t_{N-1}$ be a window of $N$ transmission opportunities at which the senders can transmit packets with the data unit $l$ to the streaming client, prior to its delivery deadline (i.e., $t_{i}<t_{D T S, l}, \forall 0 \leq i \leq N-1$ ). The transmission policy $\pi_{l}$ for data unit $l$ then forms a matrix of binary actions $a_{m i}$, for $i=0, \ldots, N-1$, and $m=1, \ldots, M$, where the row and column indices denote respectively the corresponding sender/path and transmission opportunity at which that action is undertaken. In other words, $a_{m i}=1$ signifies the transmission of a packet with the data unit $l$ by the server $m$ at transmission opportunity $t_{i}$, and $a_{m i}=0$ signifies the converse. The arrival of a packet with the data unit at the client is immediately acknowledged to every sender by sending acknowledgment packets in the backward direction on all $M$ paths. Note that sender $m$ will only send a packet with the data unit $l$ for $a_{m i}=1$ if no acknowledgment arrives at the sender by $t_{i}$ to report the correct reception of the data unit due to earlier transmissions.

\section{B. Optimization Problem}

We are interested in finding the transmission policy that minimizes the distortion experienced at the client, under constraints 
TABLE I

PARAMETERS USED in the Distributed STREAMing Algorithm

\begin{tabular}{|c|c|}
\hline$\Delta T$ & bandwidth estimation period \\
\hline$\epsilon_{F}^{m}, \epsilon_{B}^{m}$ & forward, resp. backward loss probability on path $m$ \\
\hline$B_{l}$ & size of data unit $l$ \\
\hline$t_{D T S, l}$ & tecoding deadline for data unit $l$ \\
\hline$\pi_{l}$ & vector of transmission policies for all data units \\
\hline $\boldsymbol{\pi}$ & expected distortion resulting from policy $\boldsymbol{\pi}$ \\
\hline$R_{m}(\boldsymbol{\pi})$ & expected rate on path $m$ resulting from policy $\boldsymbol{\pi}$ \\
\hline$\tilde{R}_{m}$ & estimate of the available rate on path $m$ \\
\hline$\rho_{m}\left(\pi_{l}\right)$ & expected transmission cost on path $m$ for the transmission of data unit $l$ \\
\hline$\epsilon\left(\pi_{l}\right)$ & probability that data unit $l$ is not delivered on time \\
\hline$S_{i}$ & set of data units \\
Base layer & \\
\hline
\end{tabular}

Fig. 2. Directed acyclic graph: illustration of data unit interdependencies in a typical scalable media stream (I, P, and B, respectively, represent Intra, Predicted and Bidirectional predicted frames in MPEG terminology).

given by the available bandwidth on each network path. Suppose that there are $L$ data units in the multimedia session. Let $\pi_{l}$ be the transmission policy for data unit $l \in\{1, \ldots, L\}$ and let $\boldsymbol{\pi}=\left(\pi_{1}, \ldots, \pi_{L}\right)$ be the vector of transmission policies for all $L$ data units. Any given policy vector $\pi$ induces for the multimedia session an expected distortion $D(\pi)$ and a vector of expected transmission rates $R(\boldsymbol{\pi})=\left[R_{0}(\boldsymbol{\pi}) R_{1}(\boldsymbol{\pi}) \ldots R_{M}(\boldsymbol{\pi})\right]$ on the forward channels of the network paths. We thus seek the policy vector $\boldsymbol{\pi}$ that minimizes the expected distortion $D(\boldsymbol{\pi})$ such that the expected transmission rate in the forward direction on every network path does not exceed the available bandwidth, i.e.,

$$
\begin{aligned}
\boldsymbol{\pi}^{*} & =\underset{\boldsymbol{\pi}}{\arg \min } D(\boldsymbol{\pi}), \\
\text { s.t. } R_{m}(\boldsymbol{\pi}) & \leq \widetilde{R}_{m}, \quad m=1, \ldots, M .
\end{aligned}
$$

Using the method of Lagrange multipliers, we reformulate (1) as an unconstrained optimization problem. That is, we seek the policy vector $\pi$ that minimizes the expected Lagrangian $J(\boldsymbol{\pi})=D(\boldsymbol{\pi})+\sum_{m=1}^{M} \lambda_{m} R_{m}(\boldsymbol{\pi})$ for some vector of positive Lagrange multipliers $\boldsymbol{\lambda}=\left[\lambda_{1} \lambda_{2} \ldots \lambda_{M}\right]$, and thus achieves a point on the lower convex hull of the set of all achievable distortion-rate pairs $(D(\boldsymbol{\pi}), R(\boldsymbol{\pi})) .{ }^{1}$ We solve the rate-distortion optimized distributed streaming problem in the next section, and we

${ }^{1}$ Equivalently, the set of all achievable distortion-rate $\left(D, R_{1}, \ldots, R_{M}\right)$ $(\mathrm{M}+1)$-tuples. later propose a low complexity solution based on packet classification. For the sake of clarity, we summarize the notation used in this paper in Table I.

\section{RATE-Distortion Optimized Distributed StREAMING}

\section{A. Media and Network Models}

We first overview the media and network models that we use to solve the optimization problem of distributed streaming. These models are commonly accepted and have been widely used recently for the design of optimized streaming solutions. We limit ourselves to the minimal presentation necessary to understand the algorithms proposed in this paper, and refer the reader to [10], [11] for more details.

In a streaming media system, the encoded data are packetized into data units and stored in a file on a media server. We consider here non-scalable as well as layered streams proposed by recent standardization bodies, as opposed to multiple description video coding that is generally limited to two descriptions, hence two streaming servers. All the data units in the presentation have interdependencies, which can be expressed by a directed acyclic graph, as illustrated in Fig. 2. Each node of the graph corresponds to a data unit, where an edge of the graph directed from data unit $l^{\prime}$ to data unit $l$ implies that data unit $l^{\prime}$ can be decoded only if data unit $l$ is first decoded. 


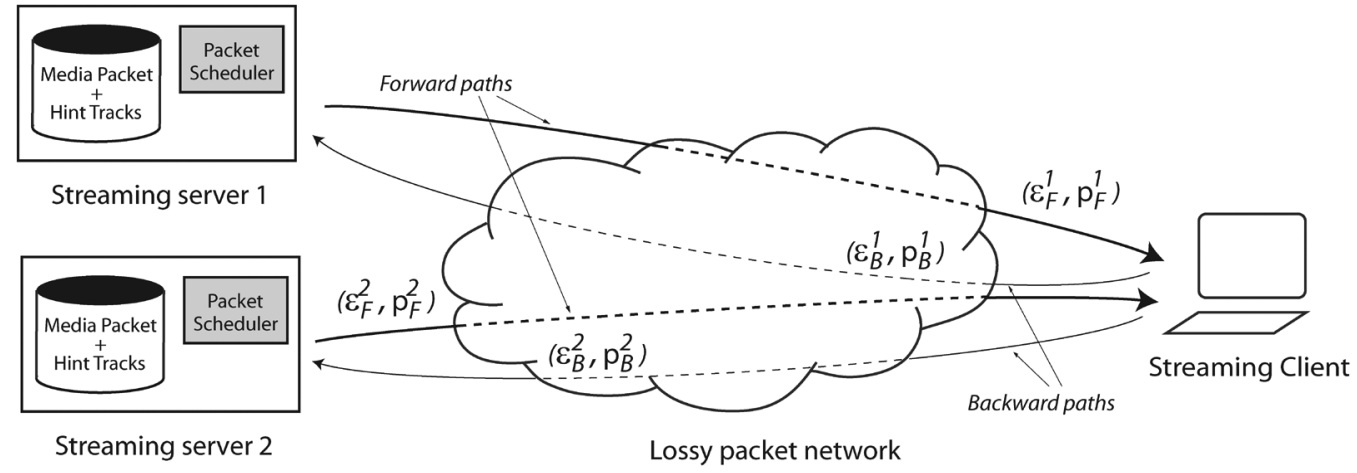

Fig. 3. Framework for distributed streaming with packet erasure channels.

Associated with each data unit $l$ is a size $B_{l}$, a decoding time $t_{D T S, l}$, a set of data units $\mathcal{N}_{c}^{(l)}$ and an importance $\Delta d_{l}^{\left(l_{1}\right)}$. Specifically, the size $B_{l}$ is the size of the data unit in bytes. $t_{D T S, l}$ is the delivery deadline by which data unit $l$ must arrive at the client to be usefully decoded. Packets containing data units that arrive after the data units' delivery deadlines are discarded. Furthermore, $\mathcal{N}_{c}^{(l)}=\{1, \ldots, l\}$ is the set of data units that the receiver considers for error concealment in case data unit $l$ is not decodable by the receiver on time. Finally, $\Delta d_{l}^{\left(l_{1}\right)}$, for $l_{1} \in \mathcal{N}_{c}^{(l)}$, is the reduction in reconstruction error (distortion) for the media presentation, when data unit $l$ is not decodable but is concealed with data unit $l_{1}$ that is received and decoded on time.

The network is represented as a simple model where the forward and backward directions on the network path between a sender (server) and the receiver are described as independent time-invariant packet erasure channels with random delay, as represented in Fig. 3. In general, each of the paths has different characteristics in terms of loss probability or latency. Hence, the path $m$ (for $m=1, \ldots, M$ ) is specified with the probabilities of packet loss $\epsilon_{F}^{m}$ and $\epsilon_{B}^{m}$, and the probability densities of the transmission delay $p_{F}^{m}$ and $p_{B}^{m}$, respectively. This means that if a server sends a packet on the forward channel $m$ at time $t$, then the packet is lost with probability $\epsilon_{F}^{m}$. However, if the packet is not lost, then it arrives at the client at time $t^{\prime}$, where the forward trip time $F T T^{m}=t^{\prime}-t$ is randomly drawn according to the probability density $p_{F}^{m}$. Therefore, we let $P\left\{F T T^{m}>\tau\right\}=\epsilon_{F}^{m}+\left(1-\epsilon_{F}^{m}\right) \int_{\tau}^{\infty} p_{F}^{m}(t) d t$ denote the probability that a packet transmitted by the server at time $t$ does not arrive at the client application by time $t+\tau$, whether it is lost in the network or simply delayed by more than $\tau$. Similar relations can be defined for the backward channel, which induce a probability $\epsilon_{R}^{m}=1-\left(1-\epsilon_{F}^{m}\right)\left(1-\epsilon_{B}^{m}\right)$ of losing a packet either on the forward or backward channel, and a round trip time distribution $P\left\{R T T^{m}>\tau\right\}=\epsilon_{R}^{m}+\left(1-\epsilon_{R}^{m}\right) \int_{\tau}^{\infty} p_{R}^{m}(t) d t$, where $p_{R}^{m}=p_{F}^{m} * p_{B}^{m}$ is the convolution of $p_{F}^{m}$ and $p_{B}^{m}$. Note that $P\left\{R T T^{m}>\tau\right\}$ is the probability that the server $m$ does not receive an acknowledgment packet by time $t+\tau$ for a data packet that has been transmitted at time $t$.

\section{B. Expected Distortion and Streaming Rate}

Now that media and network models are defined, we can compute the distortion $D(\boldsymbol{\pi})$ and the streaming rate $R(\boldsymbol{\pi})$ that result from a streaming policy $\pi$. We do that by extending to the multiple sender case the formulation proposed in [10], [11]. The expected transmission rate $R_{m}(\boldsymbol{\pi})$ on path $m$ is the sum of the expected transmission rates on this path for each data unit $l \in\{1, \ldots, L\}$ in the presentation:

$$
R_{m}(\boldsymbol{\pi})=\sum_{l} B_{l} \rho_{m}\left(\pi_{l}\right)
$$

where $B_{l}$ is the size of data unit $l$ in bytes and $\rho_{m}\left(\pi_{l}\right)$ is the expected cost per byte, or the expected number of transmitted bytes per source byte under policy $\pi_{l}$ on path $m$. The expected distortion $D(\boldsymbol{\pi})$ is somewhat more complicated to express, but it can be expressed in terms of the expected error, or the probability $\epsilon\left(\pi_{l}\right)$ that data unit $l$ does not arrive at the client on time (under policy $\pi_{l}$ ). It reads

$$
\begin{aligned}
D(\boldsymbol{\pi})=D_{0} & -\sum_{l} \sum_{l_{1} \in \mathcal{N}_{c}^{(l)}} \Delta d_{l}^{\left(l_{1}\right)} \prod_{j \in \mathcal{A}\left(l_{1}\right)}\left(1-\epsilon\left(\pi_{j}\right)\right) \\
& \times \prod_{l_{2} \in \mathcal{C}\left(l, l_{1}\right)}\left(1-\prod_{l_{3} \in \mathcal{A}\left(l_{2}\right) \backslash \mathcal{A}\left(l_{1}\right)}\left(1-\epsilon\left(\pi_{l_{3}}\right)\right)\right)
\end{aligned}
$$

where $D_{0}$ is the expected reconstruction error for the presentation if no data units are received. $\mathcal{A}\left(l_{1}\right)$ is the set of ancestors of $l_{1}$, including $l_{1} \cdot \mathcal{C}\left(l, l_{1}\right)$ is the set of data units $j \in \mathcal{N}_{c}^{(l)}: j>l_{1}$ that are not mutual descendants, i.e., for $j, k \in \mathcal{C}\left(l, l_{1}\right): j \notin$ $\mathcal{D}(k), k \notin \mathcal{D}(j)$, where $\mathcal{D}(j)$ is the set of descendants of data unit $j$, and " $\backslash$ " denotes the operator "set difference".

The expected error-cost functions for sender-driven distributed streaming can be derived by extending the results from [11] to the multiple path case. Recall that $\epsilon\left(\pi_{l}\right)$ is the probability that data unit $l$ is not delivered on time given the transmission actions in $\pi_{l}$. Furthermore, the expected cost on path $m$ at transmission opportunity $t_{i}$ is zero if $a_{m i}=0$, and otherwise it is equal to the probability that no acknowledgments arrive at sender $m$ by $t_{i}$, as a result of previous transmissions of the data unit. Hence, sender $m$ computes these two quantities using

$$
\begin{aligned}
\epsilon_{m}\left(\pi_{l}\right)= & \prod_{\substack{j<i, p: \\
a_{p j}=1}} P\left\{F T T^{p}>t_{D T S}-t_{j} \mid F T T^{p}\right. \\
& \left.+B T T^{m}>t_{i}-t_{j}\right\} \\
& \times \prod_{\substack{j \geq i, p: \\
a_{p j}=1}} P\left\{F T T^{p}>t_{D T S}-t_{j}\right\},
\end{aligned}
$$




$$
\begin{aligned}
\rho_{m}\left(\pi_{l}\right)= & \sum_{\substack{j \geq i: \\
a_{m j}=1}} \prod_{\substack{k<i, p: \\
a_{p k}=1}} P\left\{F T T^{p}+B T T^{m}>t_{j}-t_{k} \mid F T T^{p}\right. \\
& \left.\times B T T^{m}>t_{i}-t_{k}\right\} \\
& \times \prod_{\substack{i \leq k<j, p: \\
a \\
\text { a }=1}} P\left\{F T T^{p}+B T T^{m}>t_{j}-t_{k}\right\}
\end{aligned}
$$

where the conditional probabilities in (4) and (5) can be computed using Bayes' rule [12] and the fact that $P\{x>a, x+y>$ $b\}=P\{x>a\}$, for $x, y$ nonnegative and $a>b$. To this end, note that the probability densities of the sums $F T T^{p}+B T T^{m}$ can be obtained as the convolution of the corresponding densities for $F T T^{p}$ and $B T T^{m}$.

\section{Rate-Distortion Optimization}

Based on the expressions for the expected distortion and streaming rate as functions of the streaming policy $\boldsymbol{\pi}$, we now have to find the policy vector that minimizes the expected Lagrangian $J(\boldsymbol{\pi})$. This is in general difficult since the terms involving the individual policies $\pi_{l}$ in $J(\boldsymbol{\pi})$ are not independent. Therefore, we employ an iterative descent algorithm, called Iterative Sensitivity Adjustment (ISA), in which we minimize the objective function $J\left(\pi_{1}, \ldots, \pi_{L}\right)$ one variable at a time while keeping the other variables constant, until convergence [10], [11]. The appropriate choice of the Lagrange multipliers $\lambda_{m}$ that allow to meet the bandwidth constraints is then determined as follows. We initially select $\lambda_{m}=\lambda$, for $m=1, \ldots, M$, and some $\lambda>0$. We repeatedly rerun the ISA optimization algorithm till convergence, while adjusting one of the Lagrange multipliers every time the optimization algorithm converges. The Lagrange multiplier $\lambda_{m}$ is adjusted using the bisection search technique [13]-[15] until either the target rate is achieved or the interval wherein $\lambda_{m}$ can range becomes smaller than a predefined threshold. The procedure outlined above is repeated until we properly adjust all Lagrange multipliers.

It is the duty of sender $m, m=1, \ldots, M$, to recompute the optimal policy $\pi_{l}^{*}$ for data unit $l$ at every $t_{i}$ and then execute $a_{m i}$ from $\pi_{l}^{*}$. This is done in order to account for acknowledgments received by the senders due to packets carrying other data units sent prior to $t_{i}$ and also to account for prospective bandwidth variations on the network paths. Clearly, the search for the optimal transmission policy, as described before, may lead to systems that does not scale very well in practice; indeed, the overall computational complexity, as well as the computational complexity at each server, increase with the number of senders. This is the price to pay for having a fully distributed streaming strategy, where the sending servers do not communicate among themselves. In the subsequent section, we therefore propose a low complexity algorithm based on source pruning and packet classification that dramatically reduces the computational requirements of the system.

\section{LOW-COMPLEXITY DiSTRIBUTED STREAMING}

\section{A. Packet Classification Based on Source Pruning}

In order to reduce the complexity of the rate-distortion optimization problem described in the previous section, we now design a low-complexity algorithm based on media packet classification. The classification that can be computed offline, is built on a rate-distortion pruning strategy, which has been proposed in [16] for the design of low-complexity adaptive streaming systems. The source pruning strategy defines the set of the most important packets that should be transmitted when the average bit rate is constrained. It establish priorities between packets and proceeds as follows.

We are interested in finding the vector of packet selection actions $\boldsymbol{b}=\left(b_{1}, \ldots, b_{L}\right)$ for the presentation, where $b_{i}=1$ denotes the action of keeping data unit $i$ in the presentation, while $b_{i}=0$ signifies the converse. The incurred reconstruction error (or distortion) for the media presentation associated with a particular vector $\boldsymbol{b}$ is denoted $D(\boldsymbol{b})$ and can be computed as

$$
D(\boldsymbol{b})=\sum_{i=1}^{L} \Delta d_{i}\left(\mathcal{A}_{i}(\boldsymbol{b})\right)
$$

where the notation $\mathcal{A}_{i}(\boldsymbol{b})$ simply signifies the fact that the choice of a subset from $\mathcal{A}_{i}$ that will be used to reconstruct data unit $i$ depends on the selection vector $\boldsymbol{b}$. Similarly, the associated data rate of the source $R(\boldsymbol{b})$ as a function of the selection vector can be computed as $R(\boldsymbol{b})=\sum_{i=1}^{L} B_{i} b_{i}$. We are interested in finding the optimal selection vector $\boldsymbol{b}^{*}$ that minimizes the resulting reconstruction error and for which the data rate of the source does not exceed the available resource as given by $R^{*}$, i.e.,

$$
\boldsymbol{b}^{*}=\arg \min D(\boldsymbol{b}), \text { s.t. } R(\boldsymbol{b}) \leq R^{*}
$$

Using the method of Lagrange multipliers the solution to the constrained optimization problem can be replaced with an equivalent convex hull approximation that is obtained as a solution of the unconstrained optimization problem given as

$$
\boldsymbol{b}^{*}=\arg \min D(\boldsymbol{b})+\lambda R(\boldsymbol{b})
$$

where $\lambda>0$ is a Lagrange multiplier. The adjustment of $\lambda$ according to the rate constraint $R^{*}$ is performed in an iterative fashion using a bisection search technique. Due to interdependencies between media packets, the solution for the optimal policy vector is again computed by using the ISA algorithm that minimizes the Lagrangian $J(\boldsymbol{b})=D(\boldsymbol{b})+\lambda R(\boldsymbol{b})$ one component at a time, until convergence [10], [11], [16].

The source pruning algorithm leads to a straightforward classification of the media packets as a function of the target streaming rate. Running the pruning scheme at successive target rates directly results in fine packet classification. The packet partitioning then simply performs as follows. Let $R_{1}, \ldots, R_{K}$ be a sequence of $K$ monotonically increasing data rates. Packets of a media stream are classified into $K$ sets $S_{1}, \ldots, S_{K}$, where the sets $S_{i}$ are obtained by pruning the source at the corresponding rates $R_{i}$, for $i=1, \ldots, K$. It is important to note 


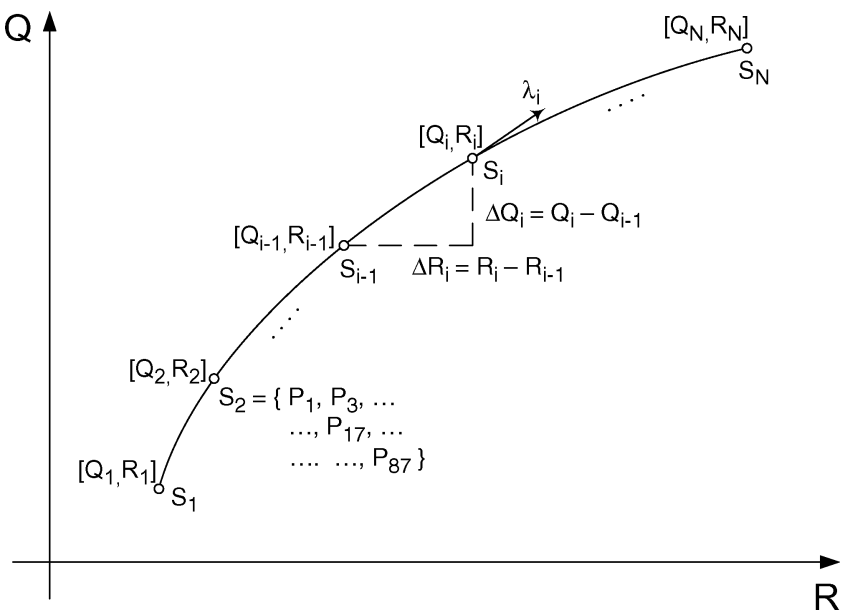

Fig. 4. Operational $R-Q$ function obtained via packet classification.

that the pruning algorithm typically creates embedded sets, ${ }^{2}$ i.e., for any two sets $S_{i}$ and $S_{j}$ such that $i<j$, it holds that $S_{i} \subset S_{j}$. Therefore, a comprehensive pruning of the full stream is not necessary at all target rates, and the classification can be performed iteratively with a limited complexity.

In essence, the procedure described above outlines the operational rate-quality ${ }^{3}$ function for the media source, as represented by its data units. This is illustrated in Fig. 4, where for each rate point $R_{i}$, there is a companion video quality point $Q_{i}$, and where the data units are denoted as packets $P_{l}$, for $l=1, \ldots, L$. The incremental increase in quality and rate that each new set $S_{i}$ provides relative to its predecessor $S_{i-1}$ can be determined as $\Delta Q_{i}=Q_{i}-Q_{i-1}, \Delta R_{i}=R_{i}-R_{i-1} \cdot{ }^{4}$ Hence, each segment $i$ of the operational $R-Q$ function can be characterized with a "gradient" of the function (on that segment) defined as $\lambda_{i}=\Delta Q_{i} / \Delta R_{i}$. In plain words, $\lambda_{i}$ denotes the per unit rate increase in quality that the segment $i$ adds to the reconstructed video stream. For more details on the packet pruning algorithm, the reader is referred to the cited [16].

\section{B. Streaming via Packet Partitioning}

We propose the following algorithm for partitioning the packets of a video stream among the $M$ senders based on the available bandwidth. Each sender employs the technique proposed above in order to create a priori the subsets $S_{i}$ for the video stream. A sufficiently large range of data rates is chosen so that it covers the possible bandwidth fluctuations encountered on the network paths. Moreover, a sufficiently large $K$ is chosen so that there is fine (incremental) division of the data rate range $R_{K}-R_{1}$. Note that $R_{K}$ should be chosen such that it corresponds to the encoding rate of the source, i.e., $S_{K}$ contains all the packets from the video stream.

Now, given the vector of estimated bandwidth values on every path $\widetilde{R}_{1}, \ldots, \widetilde{R}_{M}$ the packet partitioning algorithm proceeds as

\footnotetext{
${ }^{2}$ This is due to the fact that the actual rates associated with the sets $S_{i}$ lie on the convex hull of the operational rate-distortion function for the compressed packetized media [16].

${ }^{3}$ In this context, quality is inversely proportional to reconstruction distortion. For example, distortion can represent the MSE of the reconstructed media units, while quality can then be the PSNR of this quantity.

${ }^{4}$ It can be safely assumed that $R_{0}=Q_{0}=0$.
}

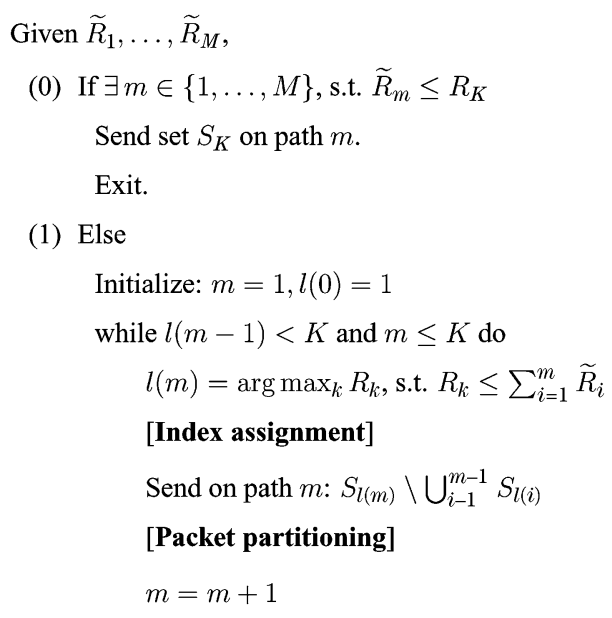

(2) End

Fig. 5. Distributed streaming via packet partitioning.

follows. If there is an $m \in\{1, \ldots, M\}$ such that it holds $\widetilde{R}_{m} \leq$ $R_{K}$, then we are done. The video stream is simply streamed on any one of the paths for which the above is true. ${ }^{5}$ Otherwise, for $m=1, \ldots, M$ each sender $m$ solves for the index $\kappa(m)$ according to

$$
\kappa(m)=\arg \max _{k} R_{k} \text {, s.t. } R_{k} \leq \sum_{i=1}^{m} \widetilde{R}_{i}
$$

and sends to the client the packets from the set $S_{\kappa(m)}$ $\bigcup_{i=1}^{m-1} S_{\kappa(i)}$, where " $"$ denotes the operator "set difference". In case there is an $m<M$ for which $\kappa(m)=K$, there is no need to run the algorithm further, i.e., for the rest of the indices $m<j \leq M$. In other words, we would reach a point where we could send all the packets from the video stream on the first $m$ paths. Note that employing such a procedure for constructing the partitions of packets sent on each path is possible because of the property that the sets $S_{i}$ are embedded, as mentioned earlier. We summarize the packet partitioning algorithm in Fig. 5.

The algorithm can be generalized in a straightforward manner to the case when the network paths exhibit packet loss in the forward direction, in addition to the varying bandwidth. In particular, let $\epsilon_{m}$ denote the erasure rate of packets sent to the client on path $m$, for $m=1, \ldots, M$. These quantities can be estimated by the client based on missing sequence numbers of arriving packets and can be piggybacked periodically to the senders on the corresponding acknowledgments. The modification on the senders' part then consists of merely employing the updated bandwidth estimates $\widetilde{R}_{1}\left(1-\epsilon_{1}\right), \ldots, \widetilde{R}_{M}\left(1-\epsilon_{M}\right)$ in the packet partitioning algorithm. Note that the effective bandwidth is surely a good approximation when the number of packets is large enough, or equivalently when the streaming rate at each server is sufficiently high.

Note that partitioning the media packets among the senders corresponds to distributing the streaming load among them based on the available bandwidth on their respective network

\footnotetext{
${ }^{5}$ For example, the senders can agree ahead of time on the strategy where the stream is sent on the first (smallest) $m$ for which this condition holds.
} 
paths. Hence, the computational complexity of packet scheduling at each server is bounded by the number of packets in the partition allocated to that server. This is equivalent to partitioning the overall search space of transmission policies for each server in the original optimization problem presented in Section III. The search space at each server decreases with the number of servers, and the computational complexity generally reduces linearly with the size of the search space [17]. Therefore, a dramatic reduction of the computational load at each server is achieved relative to the rate-distortion optimal solution.

\section{Simulation Results}

\section{A. Setup}

This section examines the performance of the proposed optimization framework, henceforth denoted RDOpt, for distributed streaming of packetized video content. The performance of its low-complexity alternative, henceforth denoted PackClas, is also investigated. The video content employed in the simulation experiments are the test video sequences Foreman and Mother \& Daughter in QCIF size encoded at $10 \mathrm{fps}$ using an H.264 codec [18]. Each sequence is encoded with a constant quantization level at an average luminance (Y) PSNR of about $36 \mathrm{~dB}$ and a Group of Pictures (GOP) size of 20 frames, where each GOP consists of an I frame followed by 19 consecutive $P$ frames. Performance is measured in terms of the average Y-PSNR of the frames of a reconstructed video content at the receiver. Video frames that are not delivered on time are replaced by the receiver using previous frame error concealment.

We consider the case with two streaming servers $(M=2)$ that transmit the video content to a client over two independent network paths. The time interval between transmission opportunities has been set to $T=100 \mathrm{~ms}$. It should be noted that at every time instance $t_{i}$ at which packet scheduling is performed, a sender considers only a subset of the media packets for the presentation. This subset is chosen by employing a sliding window over the whole set of media packets [10], according to the packets' delivery deadlines. In essence, the sliding window selects the packets that are most relevant (pressing) to be sent given the current transmission opportunity $t_{i}$.

A simple algorithm is implemented to estimate the transmission bandwidth for the streaming session. The receiving client monitors the forward-trip time (FTT) of arriving packets and piggybacks this information on returning acknowledgment packets to all the sending servers. In particular, let $F T T_{1}^{k}, F T T_{2}^{k}, \ldots, F T T_{P}^{k}$ be the transmission delays experienced by packets received by the client on path $k$ and returned to the servers via the corresponding acknowledgments in the last $\Delta T$ seconds. Then, the most recent estimate for the bandwidth (data rate) available on path $k$ is computed by the servers as $\widetilde{R}_{k}=(1 / P) \sum_{j=1}^{P}\left(B_{j} / F T T_{j}^{k}\right)$. This is simply the average of the $P$ most recent estimates of the available bandwidth on the path associated with the corresponding received packets, where $B_{j}$ is the size of packet $j$ in bits (or bytes). The time period employed at the server for bandwidth estimation has been set to $\Delta T=1 \mathrm{~s}$, in accordance to the techniques proposed in [19]. It can be noted that other bandwidth estimation methods can be implemented at the server, such as the one proposed in [19] or inspired from [20], [21], since delays and loss rate information are made available via receiver feedbacks. We do not expect that the actual bandwidth estimation method would however change the analysis and the conclusions derived in this paper.

For comparison purposes, in the simulations we also examine the performance of a conventional system, denoted Baseline, which performs proportional packet scheduling based only on the available bandwidth values. In particular, the two senders split the packets of a video stream in proportion to the bandwidth estimates on the corresponding network paths. Packet dropping decisions in Baseline are performed randomly without taking into account their specific distortion importance. In other words, Baseline is distortion-agnostic.

\section{B. Distributed Streaming Over the Internet}

We simulate the behavior of our distributed streaming system in practical settings, and we consider sending the video content over network traces of actual packet delays and losses collected in the Internet. The two senders are located on the East Coast, while the receiving destination is located on the West Coast of the continental USA. We examine the performance of the sending sources for delivering the media content to the receiving destination over their respective network paths using each of the three scheduling mechanisms considered in our experiments. To collect the network traces packet probes of 50 Bytes were sent in both directions on each path every $10 \mathrm{~ms}$ for the duration of two full days in order to sample continuously the network conditions on the paths. The data rate of the probes $(40 \mathrm{kbps})$ represents a small fraction of the access links at the sender and the receiver, thereby not having an effect on the delay and loss characteristics of the paths. For more details on the methodology that was employed to collect the traces and the related issues, the reader is referred to [22].

In Fig. 6, we examine the performance of the scheduling schemes in terms of the Y-PSNR quality computed on the sequence reconstructed at the decoder. The performances are reported as a function of the playback delay at the receiver, for streaming the Foreman sequence from two servers. It can be seen that both optimized schemes, RDOpt and PackClas, outperform the conventional system Baseline with a significant margin when the playback delay is small. This is due to the fact that RDOpt and PackClas can take advantage of the different importance of the media packets when scheduling their transmissions. In particular, by sending the most important packets first and ahead of time, these schemes increase the likelihood of successful delivery of the corresponding data units to the receiving client. This in turn reduces the reconstruction distortion for the media presentation that the client would observe on the average. On the other hand, as the baseline scheme does not have the knowledge of packet importance, it schedules every packet equally, which in turn results in higher average distortion for the reconstructed stream.

It should be mentioned that the lower end of playback delays considered here are sufficiently small (relative to the packet delays experienced on the network paths) to effectively prohibit packet retransmissions. Retransmissions would certainly improve the performance of all three scheduling mechanisms, 


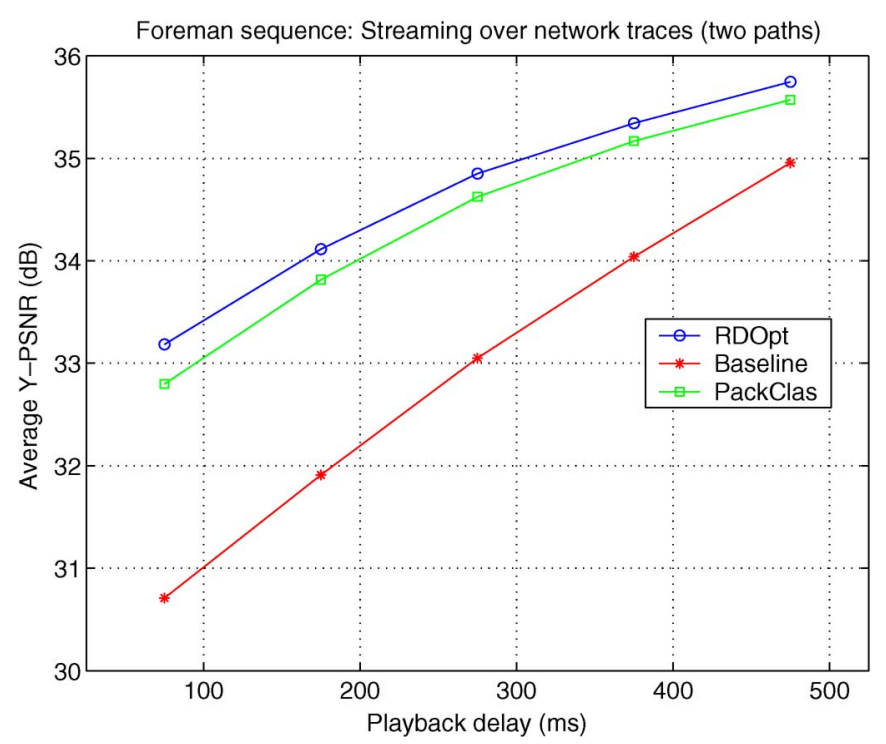

Fig. 6. Distortion performance for streaming Foreman over network traces as a function of play-out delay.

and especially of the conventional technique. This is evident from Fig. 6 for the higher end of playback delay values, when the performances of the three schemes converge. Finally, note that the difference in performance between RDOpt and PackClas is non-negligible, as shown in Fig. 6. RDOpt represents the rate-distortion bound for a given playback delay. Contrarily to PackClas, it can schedule transmissions of the same packet, in case of high importance, from both senders, which increases the likelihood of its delivery.

We observed similar relative performance between the three systems in the case of Mother \& Daughter. As seen from Fig. 7, RDOpt and PackClas again provide substantially improved performance over the baseline scheme for the lower range of playback delays. The performance difference between the three schemes then reduces as the playback delay is increased, which is expected, as argued earlier for the case of the Foreman sequence. Finally, it should be mentioned that the performance differences between the three systems exhibited in Fig. 7 are somewhat smaller relative to the corresponding ones for the Foreman sequence. This is due to the fact that error concealment works more effectively in the case of Mother \& Daughter due to the slow-moving nature of this video content. This in turn renders the presence of certain video packets less crucial for the reconstruction quality of the video presentation, thereby reducing the effects of the relative differences in packet delivery that each of the scheduling schemes may contribute to.

\section{Performance Analysis}

Here, we examine in greater detail the performance of the proposed system with a particular emphasis on its adaptivity to bandwidth variations and packet loss. We choose a simple network model in order to facilitate the analysis, where the network bandwidth in the forward direction on each path is randomly varying between a lower and an upper bound. The random fluctuations of the available bandwidth occur every two seconds.

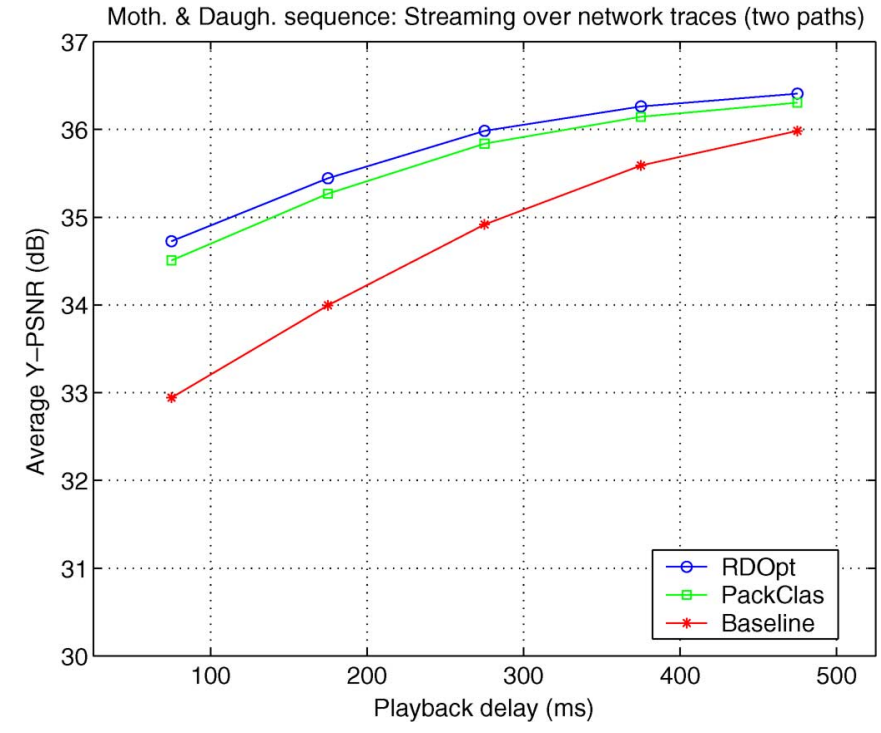

Fig. 7. Distortion performance for streaming Mother \& Daughter over network traces as a function of play-out delay.

The characterization of the bandwidth variations that we employ here has been chosen to match typical bandwidth variations observed in the Internet. At the same time, we do not expect that the performance of the distributed streaming system is highly sensitive to short time variations of the available bandwidth, as long as the play-out delay of the client application allows for buffering delays on the order of the time needed for accurate bandwidth estimation. The playback delay of the client application is set to one second, which is a small values for practical streaming systems. It moreover corresponds to the time period $\Delta T$ that is used for bandwidth estimation. In our simple network model, the packet delay densities are assumed to be exponential functions and are inherently tied to the available bandwidth on the network paths. In particular, from the M/M/1 model [23] that is frequently used to model network queues, we know that the mean of the corresponding exponential distribution for the network delay experienced by a packet is $\mu=$ PackSize $/ B W$, where PackSize $=500$ bytes is the average packet size used in our simulations, and $B W$ is the available bandwidth. The range in which the available bandwidth is randomly varying is given as $\left[B W_{\min }, B W_{\min }+20\right]$ for one of the paths, and $\left[B W_{\min }+20, B W_{\min }+40\right]$ for the other network path, where $B W_{\min }$ is measured in kbps. Hence, the paths are asymmetric in terms of available bandwidth and the backward channels that are used only for acknowledgments packets are considered to offer sufficient bandwidth in all cases.

1) Bandwidth Adaptation: We first analyze the behavior of the distributed streaming system when it faces frequent variations of the available bandwidth. In particular, the packet loss rates on the network paths are assumed to be zero (i.e., $\epsilon_{F}=$ $\epsilon_{B}=0$ ), and we study how the framework performs in adjusting the streaming rate of the video content to the bandwidth variations of the underlying network. In the simulations, we change $B W_{\min }$ across a certain range, and for each of its values we record the corresponding Y-PSNR performances of RDOpt, PackClas, and Baseline. 


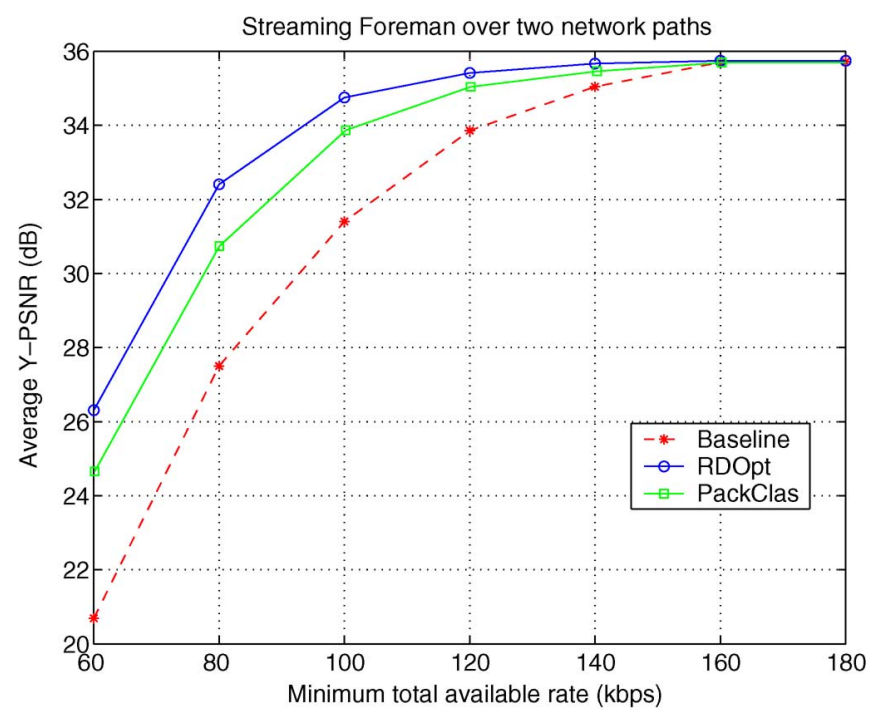

Fig. 8. Rate-distortion performance for bandwidth adaptation of Foreman.

In Fig. 8, we show the performances of the three systems under investigation for streaming the Foreman sequence, as a function of the minimum transmission bandwidth available on both paths during a session, i.e., $2 * B W_{\min }+20$. It can be seen from the figure that RDOpt provides an improved performance over the baseline system almost over the whole range of values considered for the minimum overall bandwidth that is available. The gains in performance are especially significant in the lower half of the bandwidth range. For example, at 60 kbps minimum overall bandwidth there is a difference of $4.5 \mathrm{~dB}$ in performance between the two systems. The improvement is due to the fact that the optimized system takes into account the distortion importance of the individual packets while adapting the video stream to the available bandwidth. In particular, by selecting the most important packets for transmission for the given available data rate on each path, RDOpt ensures the best possible reconstruction quality of the video presentation at the receiver. On the other hand, Baseline performs bandwidth adaptation without treating the various packets preferentially, as it is distortion-agnostic. Therefore, some more important packets may be dropped at the expense of others, less important ones, which would ultimately lead to a degradation in video quality at the client.

The low-complexity technique also outperforms the baseline system, as shown in Fig. 8. This is quite encouraging, as this system induces only a small online complexity. The improved performance of PackClas is due to the fact that the packet partitions from which the senders stream the video data are selected based on the subsets of packets $S_{i}$. These in turn are selected such that they correspond to the maximum possible video quality for the corresponding available data rates, as explained in Section IV. It should be noted though that the low-complexity technique provides a somewhat degraded performance relative to the global optimization framework. The difference in performance between the two systems reaches up to $1-1.2 \mathrm{~dB}$ in the lower end of data rates. This is anticipated, as in the former system streaming is performed based on the packet subsets $S_{i}$

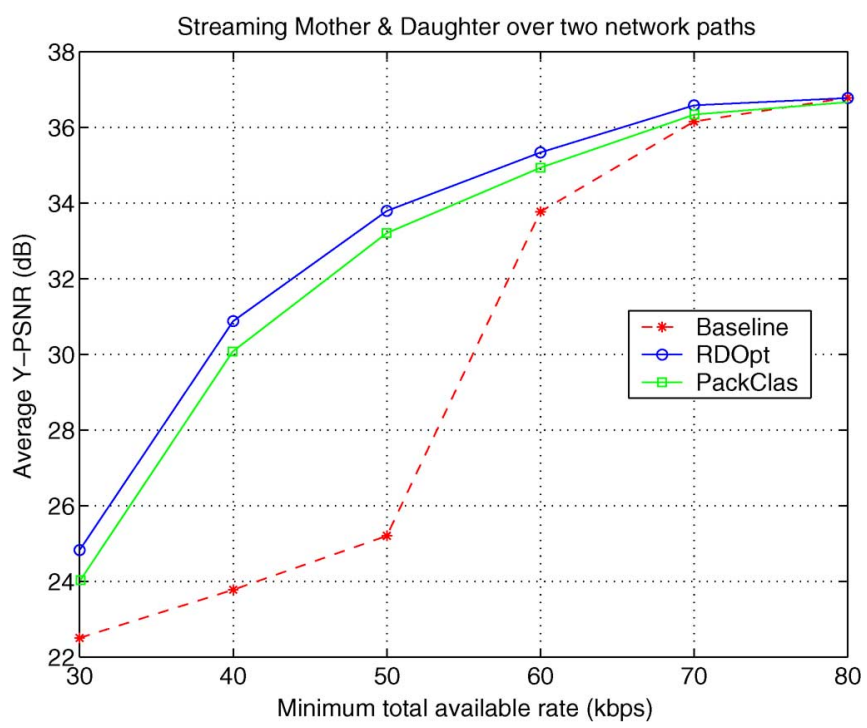

Fig. 9. Rate-distortion performance for bandwidth adaptation of Mother \& Daughter.

that are selected ahead of time. Therefore, they provide less flexibility in terms of adapting to dynamic bandwidth variations relative to the optimization framework, in which at every instance a sender has access to all the packets from a video stream when making transmission decisions. This is the necessary price that systems like PackClas pay in order to reduce their online complexity by having predefined sets of packets to choose from when streaming. The number of packet sets is finite, and therefore fine adaptation to bandwidth variations is constrained by the granularity of the $K$ target rates $\left[R_{1}, R_{2}, \ldots, R_{K}\right]$ used for the packet partitioning.

Finally, it can be seen from Fig. 8 that all three systems perform alike for sufficiently large minimum overall bandwidth. This is expected here since there is sufficient bandwidth available throughout the session to ensure timely delivery of all packets to the receiver in the case of each system. In other words, no packet needs to be dropped anymore due to a mismatch between the network bandwidth variations and the dynamically varying source encoding rate.

Similar relative performances for the three systems are observed for streaming Mother \& Daughter, as shown in Fig. 9. It can be seen that again both RDOpt and PackClass outperform Baseline almost over the whole range of bandwidth values under consideration, with gains reaching up to $6-8 \mathrm{~dB}$ in the lower half of the bandwidth range. Similarly, there is a performance gap between RDOpt and PackClass, which is due to the reason explained earlier. Finally, when the overall available bandwidth reaches a value at which no bandwidth adaptation is needed throughout the session, all three systems perform identically, as illustrated by their performances for $80 \mathrm{kbps}$ minimum overall bandwidth.

2) Adaptation to Bandwidth and Packet Loss: Next, we examine the performance of RDOpt, PackClas, and Baseline when adapting to both bandwidth variations and random packet loss. In particular, in addition to dynamic bandwidth variations the communication channels also exhibit random packet erasures. Therefore, packets will be lost during transmission 

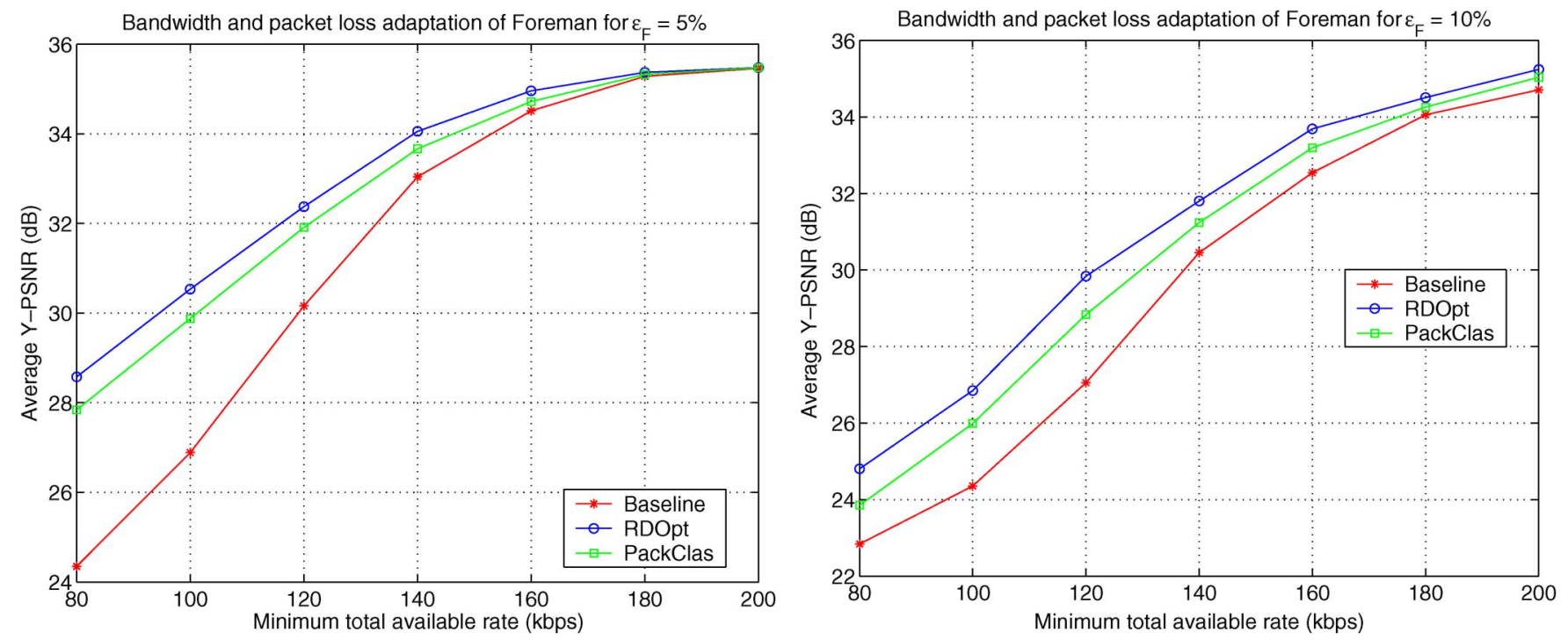

Fig. 10. Bandwidth and packet loss adaptation of Foreman: (left) $\epsilon_{F}=5 \%$ and (right) $\epsilon_{F}=10 \%$.

and a streaming system needs to decide then whether it would retransmit packets or send new packets. This trade-off is necessitated by the fact that the available bandwidth is insufficient to support sending all the packets together, including the (re)transmissions. Note that the target rates used for the packet classification in PackClas are adapted to match the effective bandwidth on the streaming paths (see Section IV-B), in order to take the loss process into account. In the new set of simulations, we measure the performances of the three systems as a function of the available data rate, but now in the presence of packet loss. Specifically, we examine packet loss rates $\left(\epsilon_{F}\right)$ of $5 \%$ and $10 \%$ on the forward channels from the senders to the receiver.

In Fig. 10, we show the performances of the three distributed streaming systems for transmitting the Foreman sequence on lossy channels. First, it can be seen that all of them exhibit a degraded performance relative to the corresponding results shown in Fig. 8, where only bandwidth adaptation is performed. This is expected since each of the streaming systems has to take into account retransmissions of lost packets, in addition to discarding packets due to bandwidth variations. Therefore, higher data rates on the communication channels are needed to achieve the same Y-PSNR performance relative to the case of bandwidth adaptation only. Second, it can also be seen that the performances of RDOpt, PackClas, and Baseline degrade with increasing the packet loss rate. For example, for $\epsilon_{F}=5 \%$, all three systems exhibit a Y-PSNR performance within the 33-34 $\mathrm{dB}$ range when the total available data rate on the channels is $140 \mathrm{kbps}$. However, that performance reduces to being in the range of 30-32 $\mathrm{dB}$ for the same data rate at packet loss rate of $10 \%$. Such a performance behavior across the three systems is also expected, as increasing the loss rate reduces the number of packets that can be delivered on time to the receiver, given a fixed play-out delay.

The two optimized systems RDOpt and PackClas provide the most significant gains over Baseline in the lower end of data rates under consideration, especially for $\epsilon_{F}=5 \%$, as seen from
Fig. 10 (left). This is expected as these systems can trade off the importance of each packet for the effective data rate, while the conventional system is distortion agnostic. However, as the packet loss rate is increased RDOpt and PackClas cannot take a lot of advantage of their knowledge of the packets' distortion importance. Even though packets are prioritized in terms of transmission, they become more likely to be lost and there is not enough data rate to perform loss recovery by retransmission. On the other hand, when there is sufficient data rate available on the channels, all three systems can deal effectively with packet losses by retransmission, as illustrated by their similar performance in the upper end of the data rate range. Note that again RDOpt outperforms PackClas over all data rates and packet loss rates considered, as shown in Fig. 8. RDOpt achieves that by taking advantage of the fact that it considers for transmission the complete set of video packets, all of the time. This in turn allows for more efficient dynamic adaption, as explained before.

In Fig. 11 we show the corresponding performances of RDOpt, PackClas, and Baseline for streaming the "Mother \& Daughter" sequence. It can be seen that the relative performances of the three systems across the different data rates and packet loss rates under consideration exhibit a similar behavior to those for the case of the Foreman sequence. In particular, the optimized systems outperform more significantly the conventional system in the lower end of data rates and especially for lower packet loss rates. As the packet loss rate increases the performances of the three systems degrade significantly and therefore become more alike. In the same spirit, as the available data rate is sufficiently large, the three systems perform alike again. In this case, there is enough bandwidth to recover (almost completely) from packet losses by retransmissions, as shown in Fig. 11.

3) Bandwidth Estimation Performance: Finally, we examine the performance of the simple algorithm described earlier for estimating the available bandwidth on a network path. In Fig. 12 (left), we show the bandwidth evolution over time on one of the network paths when streaming Foreman. The solid line denotes 

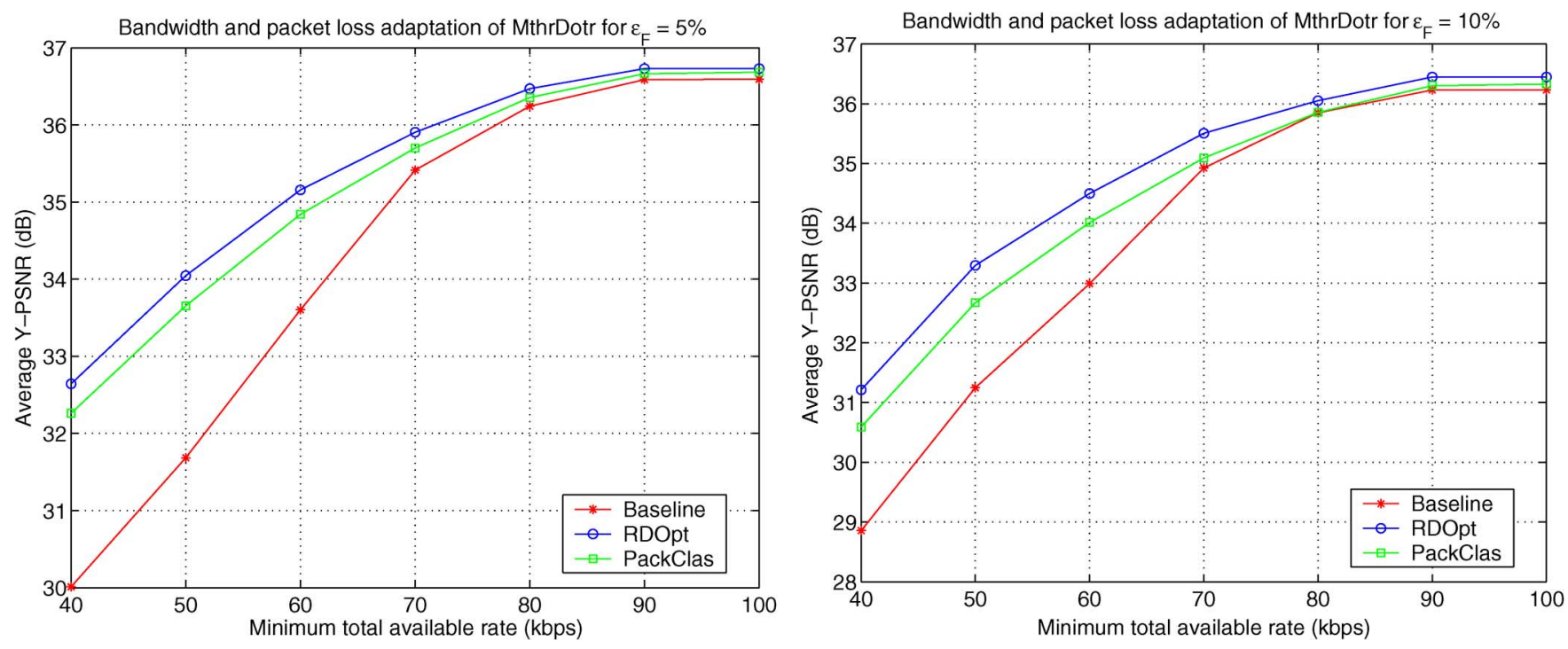

Fig. 11. Bandwidth and packet loss adaptation of Mother \& Daughter: (left) $\epsilon_{F}=5 \%$ and (right) $\epsilon_{F}=10 \%$.
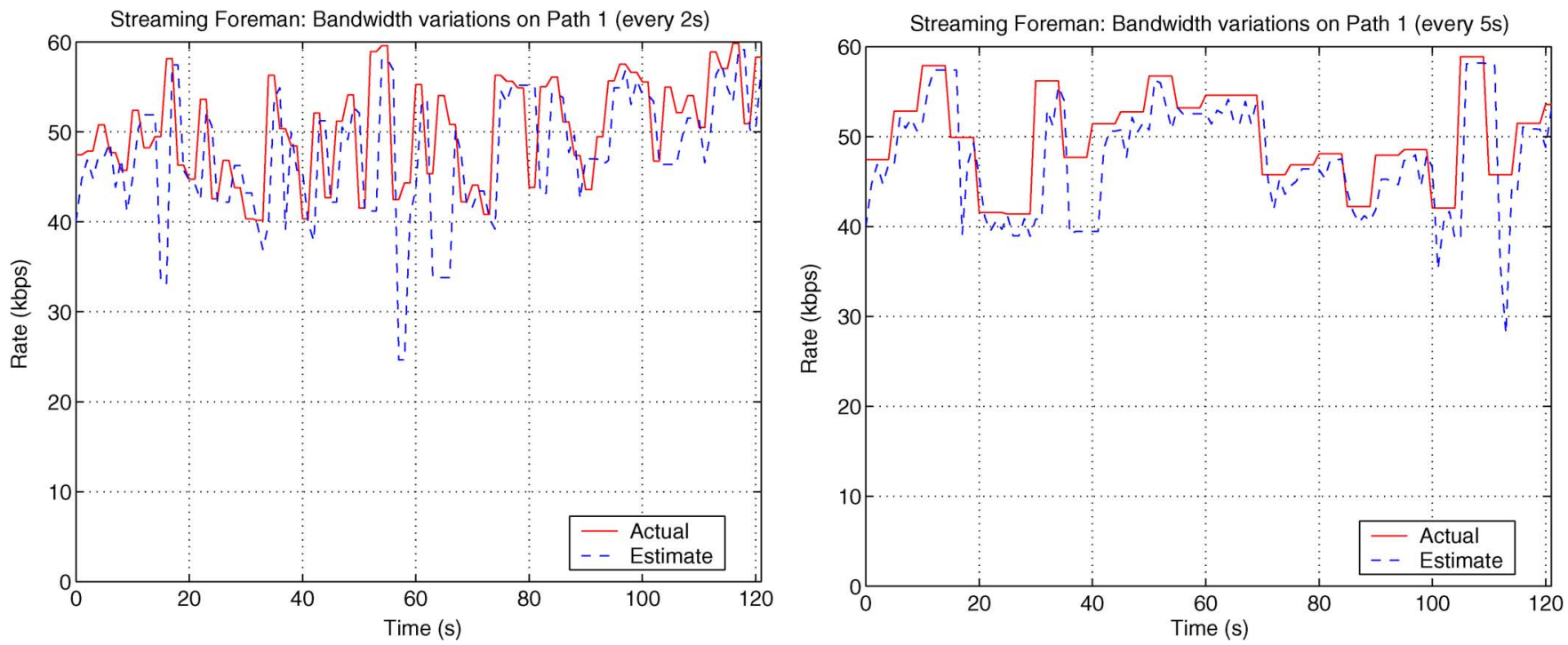

Fig. 12. Bandwidth variations over time on a network path when streaming Foreman. Random bandwidth changes occur every (left) $2 \mathrm{~s}$, (right) $5 \mathrm{~s}$.

the actual bandwidth values, while the dashed line represents its estimate obtained using the proposed algorithm. It can be seen from Fig. 12 (left) that the estimated values track quite well the dynamic variations of the actual bandwidth on the path, especially given the fact that random bandwidth changes are initiated frequently (every $2 \mathrm{~s}$ ) relative to the bandwidth estimation period (every one second). Notable discrepancies between the actual and the estimated values occur only when there is a substantial sudden reduction in the available bandwidth, as illustrated at a few points in the bandwidth trace shown in Fig. 12 (left) (for example around Time equal to $18 \mathrm{~s}$ and $60 \mathrm{~s}$ on the $\mathrm{x}$-axis). This is expected, as the procedure for estimating the available bandwidth is causal, i.e., it is based on previously received acknowledgment packets within a time period. Therefore, when the available network bandwidth suddenly and substantially drops there are not so many acknowledgment packets received within the next time period that can be used for accurate bandwidth estimation.
In order to examine how the frequency of bandwidth variations affects the accuracy of the estimation technique, we performed the same simulations as before, but now with random bandwidth fluctuations occurring every five seconds. These results are shown in Fig. 12 (right). It can be seen that the estimated bandwidth values track even closer now the actual bandwidth on a network path. That is because the bandwidth changes less frequently now relative to the estimation period, which allows for better and more stable estimates to be performed using the proposed technique, as illustrated in Fig. 12 (right).

Finally, a useful evidence for the interpretation of the streaming results presented earlier is the source encoding rate of the Foreman video sequence used in the simulations, which has an average value of $82.23 \mathrm{kbps}$. Even though the minimum overall bandwidth is $100 \mathrm{kbps}$, and the average source encoding rate is almost $20 \mathrm{kbps}$ smaller than that, still there are points along the time axis when the instantaneous source encoding rate exceeds the available network bandwidth (marked with 


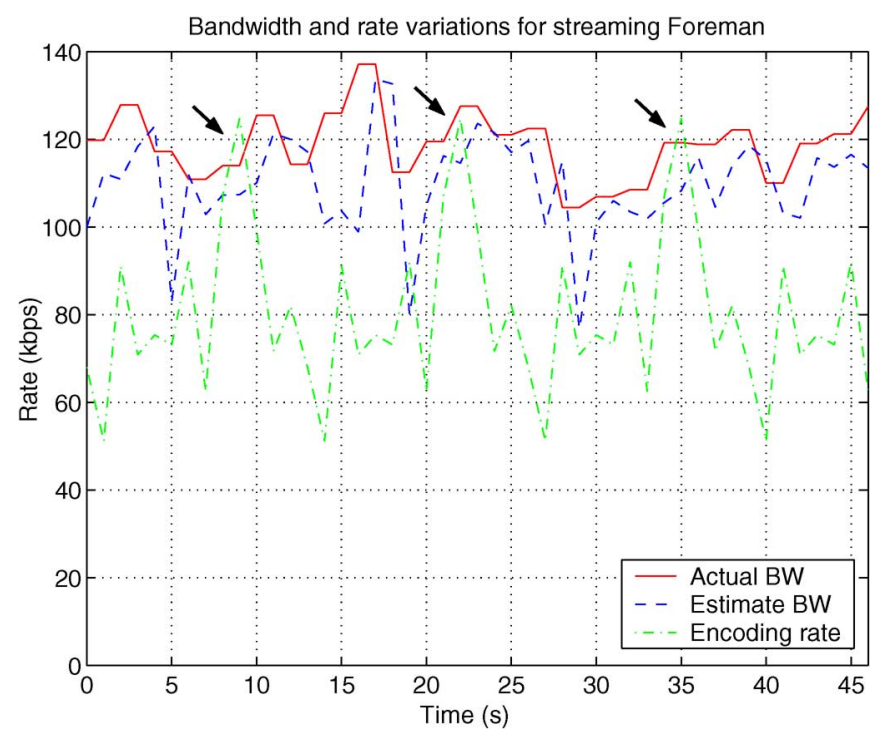

Fig. 13. Dynamic variations of overall bandwidth on the paths and of encoding rate for Foreman.

arrows in Fig. 13 at Time roughly equal to $8 \mathrm{~s}$ and $35 \mathrm{~s}$ on the $\mathrm{x}$-axis). Therefore, packets need to be dropped at these points to compensate for the insufficient transmission bandwidth, which results in loss in Y-PSNR performance. It is interesting to note that the same situation occurs when the instantaneous source rate is larger than the estimate of the available network bandwidth (though not necessarily of its actual value), as evident from Fig. 13 at Time equal to roughly $22 \mathrm{~s}$. This is exactly where the packet prioritization performed by the optimized distributed streaming solutions proves to be advantageous compared to a distortion-agnostic schedulers. Lastly, it should be pointed out that analogous observations regarding the dynamics of the source rate and the available network bandwidth were made for the case of streaming Mother \& Daughter.

\section{Discussion}

We have shown that the distributed streaming system based on packet classification provides performance that is quite competitive with the rate-distortion optimal solution. The suboptimal behavior is due to several design choices, which objectives are mainly driven by reducing the computational complexity of the optimal streaming solution. In particular, a priori partitioning of the media packets breaks the dependencies between media units, since each server manages a distinct subset of data units, independently of the streaming strategies decided by other servers, on other complementary subsets of data units. Additionally, we observed in our simulations that the rate-distortion optimal strategy sometimes sends the same packet from different servers in order to increase the probability of on-time arrival at the client of important packets. Such a strategy is excluded from the streaming policies available in PackClas scheme, due to the partitioning of the media packets into distinct subsets. Finally, another limitation of PackClas stems from the granularity of target rates when classification of the media packet is performed originally (see Section IV-B). For computational complexity reasons, the granularity cannot be chosen arbitrarily fine, which in turn affects how efficient PackClas can be when adapting to bandwidth variations. Nonetheless, PackClas proves to be a competitive solution for distributed streaming applications thanks to its low computational complexity, ease of deployment due to minimal requirements on the receiver, and yet efficient rate-distortion performance.

\section{CONCLUSIONS}

We presented a system for rate-distortion optimized packet scheduling in distributed video streaming scenarios where several sources collaborate to serve a media client. The system consists of an optimization framework for scheduling the packet transmissions at the individual senders. Using bandwidth estimates based on client feedbacks, the senders can independently, but still in coordination, decide what the most important packets are to transmit on every network path, for the given bandwidth estimates. We designed a low-complexity solution that precomputes optimized packet schedules ahead of time thereby reducing substantially the required online complexity during streaming. This is achieved by employing packet classification, via source pruning, of the compressed video stream at different data rates. Extensive simulations demonstrate minimal performance loss compared to the complex rate-distortion optimal solution, as well as significant performance gains over a conventional distortion-agnostic system for distributed streaming with comparable online complexity.

\section{ACKNOWLEDGMENT}

The authors would like to thank Dr A. Markopoulou for providing the Internet traces [22] used in the simulations.

\section{REFERENCES}

[1] J. Byers, M. Luby, and M. Mitzenmacher, "Accessing multiple mirror sites in paralel: Using tornado codes to speed up downloads," in Proc. Conf. on Computer Communications (INFOCOM), New York, Mar. 1999, vol. 1, pp. 275-283, IEEE.

[2] S. Savage, A. Collins, E. Hoffman, J. Snell, and T. Anderson, "The end-to-end effects of internet path selection," in Proc. Data Communication, Ann. Conf. Series, Cambridge, MA, Aug. 1999, pp. 289-299, ACM.

[3] J. Apostolopoulos, T. Wong, W.-T. Tan, and S. Wee, "On multiple description streaming with content delivery networks," in Proc. Infocom, New York, June 2002, vol. 3, pp. 1736-1745, IEEE.

[4] T. Nguyen and A. Zakhor, "Distributed video streaming over internet," in Proc. Multimedia Computing and Networking, San Jose, CA, Jan. 2002, vol. 4673, pp. 186-195, SPIE.

[5] T. Nguyen and A. Zakhor, "Distributed video streaming with forward error correction," in Proc. Int. Packet Video Workshop, Pittsburgh, PA, Apr. 2002.

[6] A. Majumdar, R. Puri, and K. Ramchandran, "Distributed multimedia transmission from multiple servers," in Proc. Int. Conf. Image Processing, Rochester, NY, Sept. 2002, vol. 3, pp. 177-180, IEEE.

[7] J. Kim, R. M. Mersereau, and Y. Altunbasak, "Network-adaptive video streaming using multiple description coding and path diversity," in Proc. Int Conf. Multimedia and Exhibition, Baltimore, MD, July 2003, vol. 2, pp. 653-656, IEEE.

[8] J. Chakareski and B. Girod, "Server diversity in rate-distortion optimized streaming of multimedia," in Proc. IEEE Int. Conf. Image Processing, Barcelona, Spain, Sept. 2003, vol. 3, pp. 645-648.

[9] J. Apostolopoulos, W.-T. Tan, and S. Wee, "Performance of a multiple description streaming media content delivery network," in Proc. IEEE Int. Conf. Image Processing, Rochester, NY, Sep. 2002, vol. 2, pp. 189-192.

[10] P. A. Chou and Z. Miao, Rate-Distortion Optimized Streaming of Packetized Media Microsoft Research, Redmond, WA, Tech. Rep. MSR-TR-2001-35, Feb. 2001. 
[11] J. Chakareski and B. Girod, "Rate-distortion optimized packet scheduling and routing for media streaming with path diversity," in Proc. Data Compression Conf., Snowbird, UT, Mar. 2003, pp. 203-212, IEEE Computer Society.

[12] A. Papoulis and S. Unnikrishna Pillai, Probability, Random Variables and Stochastic Processes, 4th ed. New York: McGraw-Hill, 2001.

[13] Y. Shoham and A. Gersho, "Efficient bit allocation for an arbitrary set of quantizers," IEEE Trans. Acoust. Speech, Signal Process., vol. 36, no. 1, pp. 1445-1453, Sep. 1988.

[14] K. Ramchandran and M. Vetterli, "Best wavelet packet bases in a rate-distortion sense," IEEE Trans. Image Process., vol. 2, no. 2, pp. 160-175, Apr. 1993.

[15] G. M. Schuster, G. Melnikov, and A. K. Katsaggelos, "A review of the minimum maximum criterion for optimal bit allocation among dependent quantizers," IEEE Trans. Multimedia, vol. 1, no. 1, pp. 3-17, Mar. 1999.

[16] J. Chakareski and P. Frossard, "Low-complexity adaptive streaming via optimized a priori media pruning," in Proc. Workshop on Multimedia Signal Processing, Shanghai, China, Oct./Nov. 2005, IEEE.

[17] C. D. Vleeschouwer, J. Chakareski, and P. Frossard, "The virtue of patience in low complexity scheduling of packetized media with feedback," IEEE Trans. Multimedia, vol. 9, no. 2, pp. 348-365, Feb. 2007.

[18] Telecom. Standardization Sector of ITU, Video Coding for Low Bitrate Communication Draft ITU-T Recommendation H.264, Mar. 2003.

[19] V. Ribeiro, R. Riedi, R. Baraniuk, J. Navratil, and L. Cottrell, "pathChirp: Efficient available bandwidth estimation for network paths," in Proc. 4th Passive and Active Measurements Workshop, La Jolla, CA, Apr. 2003.

[20] S. Floyd, M. Handley, J. Padhye, and J. Widmer, "Equation-based congestion control for unicast applications," in Proc. Data Communication, Ann. Conf. Series, Stockholm, Sweden, Aug. 2000, pp. 43-56, ACM.

[21] J. Padhye, J. Kurose, D. Towsley, and R. Koodli, “A TCP-friendly rate adjustment protocol for continuous media flows over best effort networks," in Proc. Int. Conf. on Measurement and Modeling of Computer Systems, Atlanta, Jun. 1999, vol. 27, pp. 220-221, ACM.

[22] A. Markopoulou, F. Tobagi, and M. Karam, "Assessing the quality of voice communications over Internet backbones," IEEE/ACM Trans. Networking, vol. 11, no. 5, pp. 747-760, Oct. 2003.

[23] D. Bertsekas and R. Gallager, Data Networks, 2nd ed. Englewood Cliffs, N.J.: Prentice-Hall, 1992.

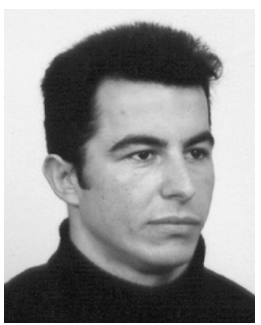

Jacob Chakareski received the B.S. degree from Ss. Cyril and Methodius University, Skopje, Macedonia. He carried out his M.S. studies at the Worcester Polytechnic Institute, in Worcester, MA. He was a Ph.D. student at Rice University, Houston, TX, and at Stanford University, Palo Alto, CA. His degrees are in electrical and computer engineering.

In 2005-2006, he was a Postdoctoral Researcher with the Swiss Federal Institute of Technology (EPFL), Lausanne. In 2006-2007, he was a Senior Engineer with Vidyo, Inc., Hackensack, NJ, where he worked on real-time video communication. He has held research positions with Microsoft and Hewlett-Packard. He has co-authored over 50 international publications and has several pending or approved patent applications. His fields of interest are networked media systems, distributed computation and control, and wireless communication.

Dr. Chakareski received the best student paper award at the IS\&T/SPIE VCIP 2004 conference.

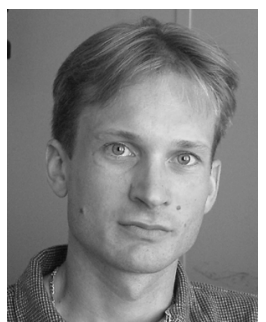

Pascal Frossard (S'96-M'01-SM'04) received the M.S. and Ph.D. degrees, both in electrical engineering, from the Swiss Federal Institute of Technology (EPFL), Lausanne, Switzerland, in 1997 and 2000, respectively.

Between 2001 and 2003, he was a member of the research staff at the IBM T. J. Watson Research Center, Yorktown Heights, NY, where he worked on media coding and streaming technologies. Since 2003, he has been an Assistant Professor at EPFL, where he heads the Signal Processing Laboratory (LTS4). His research interests include image representation and coding, nonlinear representations, visual information analysis, joint source and channel coding, multimedia communications, and multimedia content distribution.

Dr. Frossard has been the General Chair of IEEE ICME 2002 and Packet Video 2007, and a member of the organizing or technical program committees of numerous conferences. He has been an Associate Editor of the IEEE TRANSACTIONS ON MUlTiMEDIA (2004-) and of the IEEE TRANSACTIONS ON CiRCUITS AND SYSTEMS FOR VIDEO TEChNOLOGY (2006-). He is an elected member of the IEEE Image and Multidimensional Signal Processing Technical Committee (2007-), the IEEE Visual Signal Processing and Communications Technical Committee (2006-), and the IEEE Multimedia Systems and Applications Technical Committee (2005-). He has served as Vice-Chair of the IEEE Multimedia Communications Technical Committee (2004-2006) and as a member of the IEEE Multimedia Signal Processing Technical Committee (2004-2007). He received the Swiss NSF Professorship Award in 2003, and the IBM Faculty Award in 2005. 